\title{
Preparación y caracterización de cementos belíticos blancos activados con dopantes alcalinos
}

\section{Preparation and characterization of alkali-activated white belite cements}

K. Morsli(*,**), Ángeles G. de la Torre(*), Antonio J. M. Cuberos ${ }^{(*)}$, Mohammed Zahir ${ }^{(* *)}$, Miguel A. G. Aranda ${ }^{(*)}$

\section{RESUMEN}

Se han preparado clínkeres blancos activados con un porcentaje en peso teórico de belita (silicato dicálcico) del $60 \%$, a una temperatura $100{ }^{\circ} \mathrm{C}$ menor que los clínkeres Portland blancos convencionales. El proceso de activación se ha llevado a cabo añadiendo a las mezclas crudas cantidades variables de $\mathrm{K}_{2} \mathrm{CO}_{3} \circ \mathrm{Na}_{2} \mathrm{CO}_{3}$. Utilizando la difracción de rayos- $X$ y la metodología de Rietveld se ha comprobado la estabilización a temperatura ambiente de porcentajes variables de los polimorfos de alta temperatura de la belita por la adición de alcalinos. De hecho, al añadir $2,0 \%$ en peso de $\mathrm{Na}_{2} \mathrm{O}$ se ha obtenido $16,4(4) \%$ de $\alpha-\mathrm{C}_{2} \mathrm{~S}, 16,7(8) \%$ de $\alpha^{\prime}{ }^{-} \mathrm{C}_{2} \mathrm{~S}$, $23,2(6) \%$ de $\beta-C_{2} S, 32,7(7) \%$ de $C_{3} S$ total, $9,5(2) \%$ de $C_{3} A$ ortorrómbico y $1,50(5) \%$ de cal libre, porcentajes en peso. Se ha comprobado que el grado de volatilización de los alcalinos, en estas condiciones de preparación, ha sido muy acusado para el $\mathrm{K}_{2} \mathrm{O}$, lo que ha provocado una menor estabilización de los polimorfos tipo- $\alpha-C_{2} S$. Además, se ha realizado un estudio calorimétrico para correlacionar el ensamblaje de fases con el comportamiento de hidratación a edades tempranas.

Palabras clave: cementos belíticos blancos activados, difracción de rayos- $X$, método de Rietveld, calorimetría isoterma de conducción.

\section{SUMMARY}

Activated white cement clinkers with a theoretical $60 \%$ (wt) belite (dicalcium silicate) content were prepared at a temperature $100{ }^{\circ} \mathrm{C}$ lower than used to manufacture conventional white Portland cement clinkers. Activation was achieved by adding variable amounts of $\mathrm{K}_{2} \mathrm{CO}_{3}$ or $\mathrm{Na}_{2} \mathrm{CO}_{3}$ to the raw mixes. Rietveld quantitative analysis of $X$-ray diffraction findings showed that adding the alkalis stabilized the high temperature belite polymorphs. When $2.0 \%$ (wt) of $\mathrm{Na}_{2} \mathrm{O}$ was added, the composition (by wt) found was 16.4(4)\% $\alpha-C_{2} S, 16.7(8) \% \alpha^{\prime}{ }^{-} C_{2} S$, $23.2(6) \% \quad \beta-C_{2} S, \quad 32.7(7) \%$ total $C_{3} S, \quad 9.5(2) \%$ orthorhombic $C_{3} A$ and $1.50(5) \%$ free lime. Due to the high volatilization rate of $\mathrm{K}_{2} \mathrm{O}$ under the experimental conditions, it stabilized the alpha-type $C_{2} S$ less effectively than $\mathrm{Na}_{2} \mathrm{O}$. A calorimetric study was likewise performed to correlate the phase composition with early age hydration (behaviour).

Keywords: activated white belite cements, X-ray diffraction, Rietveld method, isothermal conduction calorimetry.

\footnotetext{
(*) Universidad de Málaga (Málaga, España).

(**) Université Chouaib Doukkali (El Jadida 24000, Marruecos).
} 


\section{INTRODUCCIÓN}

La actual preocupación por el cuidado del medio ambiente y el desarrollo de un sector de la construcción sostenible en general, y en particular, la necesidad de disminuir las emisiones de $\mathrm{CO}_{2}$ conlleva la búsqueda de procesos y/o materiales alternativos $(1,2)$. Una alternativa que puede contribuir a disminuir las emisiones de $\mathrm{CO}_{2}$ en un $\sim 10 \%$ son los cementos belíticos. Sin embargo la gran desventaja inherente a los clínkeres ricos en belita es la lenta reactividad con el agua, por ello es esencial activar estos materiales para mejorar la reactividad y con ella el desarrollo de resistencias a edades tempranas (3, 4). El cemento Portland blanco es uno de los cementos llamados decorativos y tiene un papel estético muy importante, pero su uso es muy limitado. Esto es debido a su elevado coste de producción, ya que algunas materias primas usadas son escasas y caras, por la exigencia de ser pobres en elementos cromóforos como el hierro, el manganeso, el cromo, etc. Además, su consumo energético en la clinkerización es elevado debido a la ausencia del hierro lo que hace que la aparición de la fase líquida sea a una temperatura mayor y la cantidad de esta fase sea menor que en el cemento gris. En la búsqueda de cementos blancos cuyos costes energéticos y económicos sean más bajos, y con propiedades específicas como la sulforresistencia, se han desarrollado nuevos materiales como son los cementos mineralizados (5) o los cementos belíticos blancos (6).

Para que los cementos ricos en belita sean utilizados de forma masiva deben ser activados (7). Uno de los caminos para conseguir la activación es estabilizar las formas polimórficas de alta temperatura de la belita (8-10). El $\mathrm{C}_{2} \mathrm{~S}$ estequiométrico presenta cinco formas polimórficas siendo la forma $\beta$, la que está presente en los cementos Portland ordinarios (CPO), aportando, sus productos de hidratación, resistencias a partir de los 28 días de curado (11). Las formas $\alpha$ son más reactivas que el resto y por ello es conveniente su estabilización a temperatura ambiente. La presencia de alcalinos, azufre o bario, y el enfriamiento rápido en el intervalo de $1.300^{\circ} \mathrm{C}$ a $900^{\circ} \mathrm{C}$ favorece la estabilización de las formas $\alpha$ (8-12).

El objetivo de este trabajo ha sido la preparación cementos belíticos blancos activados con óxidos de sodio o potasio.

\section{SECCIÓN EXPERIMENTAL}

\subsection{Preparación de materiales}

Se ha preparado un clínker belítico blanco (BB_Ref) de laboratorio, sin activar, utilizando como materias primas calcita (99\% Aldrich), $\mathrm{SiO}_{2}(99,59 \%$ ABCR) y caolín

\section{INTRODUCTION}

Today's concern about the environment, along with the furtherance of sustainable construction in general and the need to reduce $\mathrm{CO}_{2}$ emissions in particular, have led to the pursuit of alternative processes and/or materials $(1,2)$. One approach that may contribute to reducing $\mathrm{CO}_{2}$ emissions by $\sim 10 \%$ is the use of belite cements. The chief drawback to belite-rich clinkers, however, is their low reactivity with water, which must be heightened to favour the development of early age strength $(3,4)$. White Portland cement, one of the socalled decorative cements, plays an important aesthetic role in construction. Its use has been limited, however, by high productions costs resulting from the need for scarce and expensive prime materials with a low proportion of chromophorous elements such as iron, manganese, chrome and so on. Moreover, the energy demand involved in clinkerizing white cement is high, for in the absence of iron the liquid phase is reached at higher temperatures and is less abundant than in the case of grey cement. The pursuit of less costly white cements with lower energy demands and specific properties such as sulphate resistance has led to the development of new materials such as mineralized (5) or white belite cements (6).

Belite-rich cements are only apt for mass use if they are activated (7). One of the ways to achieve activation is to stabilize their high temperature belite polymorphs (8-10). Stoichiometric $C_{2} S$ exists in five polymorphic forms: the $\beta$ form is the origin of the hydration products that ensure high 28-day strength in ordinary Portland cements (OPC) (11). Stabilization at ambient temperature is sought particularly for the $\alpha$ forms, the most reactive of the polymorphs. Such stabilization is favoured by the presence of alkalis, sulphur or barium and rapid cooling from 1,300 to $900{ }^{\circ} \mathrm{C}(8-12)$.

The present study aimed to prepare white belite cements activated with sodium or potassium oxides.

\section{EXPERIMENTAL}

\subsection{Material preparation}

A non-activated white belite laboratory clinker (BB_Ref) was prepared from (99\% Aldrich) calcite (99.59\% $A B C R$ ) $\mathrm{SiO}_{2}$, and kaolin (Aldrich). The theoretical 
(Aldrich). La composición mineralógica teórica de este clínker es $60 \%$ de $\mathrm{C}_{2} \mathrm{~S}, 26 \%$ de $\mathrm{C}_{3} \mathrm{~S}$ y $14 \%$ de $\mathrm{C}_{3} \mathrm{~A}$ (\% en peso). Por otro lado se sintetizaron cuatro clínkeres blancos activados añadiendo pequeñas cantidades de $\mathrm{K}_{2} \mathrm{O}$ o $\mathrm{Na}_{2} \mathrm{O}$ en forma de $\mathrm{K}_{2} \mathrm{CO}_{3}$ (99\% Aldrich) y $\mathrm{Na}_{2} \mathrm{CO}_{3}$ $(99,999 \%$ Aldrich), respectivamente. En la Tabla 1 se dan las dosificaciones empleadas para sintetizar cada clínker expresadas en forma de óxidos. El módulo de saturación para esta familia de clínkeres es 79,5. mineralogical composition of this clinker was $60 \% \mathrm{C}_{2} S$, $26 \% C_{3} S$ and $14 \% C_{3} A$ (wt). In addition, four white clinkers were prepared and activated by adding small amounts of $\mathrm{K}_{2} \mathrm{O}$ or $\mathrm{Na}_{2} \mathrm{O}$ in the form of (99\% Aldrich) $\mathrm{K}_{2} \mathrm{CO}_{3}$ and (99.999\% Aldrich) $\mathrm{Na}_{2} \mathrm{CO}_{3}$. Table 1 gives the doses used to synthesize each clinker, expressed as oxides. The lime saturation factor (LSF) for this family of clinkers is 79.5.

Tabla 1 / Table 1

Dosificación nominal de los crudos preparados para sintetizar los clínkeres belíticos blancos expresados en forma de óxidos. El porcentaje de activadores se ha puesto en negrita.

White belite clinker síntesis: nominal dosage used in raw mixes, expressed in oxide percentage. Activator percentage shown in boldface type.

\begin{tabular}{|c|c|c|c|c|c|c|c|c|c|c|}
\hline & $\begin{array}{c}\mathbf{C a O} \\
\mathbf{\%}\end{array}$ & $\begin{array}{c}\mathbf{S i O}_{\mathbf{2}} \\
\mathbf{\%}\end{array}$ & $\begin{array}{c}\mathbf{A l}_{\mathbf{2}} \mathbf{O}_{\mathbf{3}} \\
\mathbf{\%}\end{array}$ & $\begin{array}{c}\mathbf{F e}_{\mathbf{2}} \mathbf{O}_{\mathbf{3}} \\
\mathbf{\%}\end{array}$ & $\begin{array}{c}\mathbf{5} \\
\mathbf{5}\end{array}$ & $\begin{array}{c}\mathbf{K}_{\mathbf{2}} \mathbf{O} \\
\mathbf{\%}\end{array}$ & $\begin{array}{c}\mathbf{N a}_{\mathbf{2}} \mathbf{O} \\
\mathbf{\%}\end{array}$ & $\begin{array}{c}\mathbf{M g O} \\
\mathbf{\%}\end{array}$ & $\begin{array}{c}\mathbf{S r O} \\
\mathbf{\%}\end{array}$ & $\begin{array}{c}\mathbf{P}_{\mathbf{2}} \mathbf{O}_{\mathbf{5}} \\
\mathbf{\%}\end{array}$ \\
\hline BB_Ref & 66.53 & 27.61 & 5.25 & 0.07 & 0.01 & 0.22 & 0.13 & 0.06 & 0.09 & 0.03 \\
\hline BB_1.0K & 66.00 & 27.39 & 5.21 & 0.07 & 0.01 & $\mathbf{1 . 0 1}$ & 0.13 & 0.06 & 0.09 & 0.03 \\
\hline BB_2.0K & 65.33 & 27.11 & 5.16 & 0.07 & 0.01 & $\mathbf{2 . 0 1}$ & 0.13 & 0.06 & 0.09 & 0.03 \\
\hline BB_1.0N & 65.94 & 27.37 & 5.21 & 0.07 & 0.01 & 0.22 & $\mathbf{1 . 0 0}$ & 0.06 & 0.09 & 0.03 \\
\hline BB_2.0N & 65.29 & 27.09 & 5.15 & 0.07 & 0.01 & 0.22 & $\mathbf{2 . 0 0}$ & 0.06 & 0.08 & 0.03 \\
\hline
\end{tabular}

Para la síntesis de los clínkeres se prepararon los crudos correspondientes pesando las cantidades adecuadas de materias primas y se homogeneizaron a mano en un mortero de ágata con alcohol etílico y se secaron en una estufa a $60{ }^{\circ} \mathrm{C}$. El proceso de homogeneización del crudo se realizó por triplicado. Los crudos se prensaron ( $150 \mathrm{MPa}$ ) en forma de pastillas cilíndricas para mejorar la reactividad. Las pastillas se introdujeron en crisoles de Pt/Au y se llevaron hasta $900{ }^{\circ} \mathrm{C}$ a una velocidad de calentamiento de $5^{\circ} \mathrm{C} / \mathrm{min}$, a esta temperatura se realizó un tratamiento isotermo durante 30 minutos. Posteriormente, se continuó calentando a la misma velocidad hasta $1.450{ }^{\circ} \mathrm{C}$, realizándose otra isoterma durante 15 minutos, tras lo cual se realizó un templado con corriente de aire.

Se realizó un análisis químico por espectroscopía de emisión atómica con fuente de plasma acoplado por inducción (ICP-AES) en un espectrómetro IRIS Advantage (Termo Jarrel Ash) del caolín utilizado para la preparación de los clínkeres belíticos, determinándose un pequeño porcentaje de óxidos alcalinos en esta materia prima. Estos valores se tuvieron en cuenta al añadir los carbonatos de alcalinos para propiciar la activación. Por otro lado, el contenido de óxidos alcalinos que permanece en los clínkeres se cuantificó mediante espectroscopía de emisión de llama en un espectrómetro AAnalyst 800 (Perkin-Elmer).

\subsection{Difracción de rayos- $X$ de laboratorio de polvo (DRXLP) y el método de Rietveld}

Los datos de difracción de rayos- $X$ de polvo usando radiación $\mathrm{CuK} \alpha_{1}(1,5406 \AA)$ se registraron en los Servicios
The clinkers were synthesized from the respective raw mixes, weighing the appropriate amounts of raw materials which were then blended by hand in an agate mortar with ethyl alcohol and oven-dried at $60^{\circ} \mathrm{C}$. This blending process was repeated three times. The raw mixes were pressed ( $150 \mathrm{MPa}$ ) into cylindrical pellets for enhanced reactivity. The pellets were placed in a Pt/Au crucible and heated at a rate of $5{ }^{\circ} \mathrm{C} / \mathrm{min}$ to $900^{\circ} \mathrm{C}$. After 30 minutes, they were ramped to from 900 to $1,450{ }^{\circ} \mathrm{C}$ also at $5{ }^{\circ} \mathrm{C} / \mathrm{min}$, heated for 15 minutes and cooled under a flow of air.

Inductively coupled plasma emission spectroscopy (ICP$A E S$ ), conducted on an IRIS Advantage (Termo Jarrel Ash) spectrometer, revealed a small percentage of oxides in the kaolin used to prepare the belite clinkers. These values were taken into consideration when adding the alkaline carbonate activators. The alkaline oxides remaining in the clinkers were also quantified via atomic absorption spectrometry on a Perkin-Elmer Analyst 800 spectrometer.

\subsection{Laboratory powder X-ray diffraction (LPXRD) and the Rietveld method}

Powder X-ray diffraction with CuKo $\alpha_{1}(1.5406 \AA)$ radiation was conducted at the University of Malaga's Centralized 
Centralizados de Apoyo a la Investigación (SCAI) de la Universidad de Málaga usando un difractómetro PANalytical X'Pert PRO MPD que consta de cargador automático y portamuestras giratorio. Este equipo tiene un monocromador primario del tipo Johansson, cristal de Ge (111), que proporciona una radiación estrictamente monocromática $\mathrm{CuK} \alpha_{1}$. Las rendijas de divergencia y antidivergencia se fijaron a $1 / 2$ de grado y se utilizaron rendijas Soller (haz incidente y difractado) de 0,04 rad. El sistema de detección consiste en un X'Celerator RTMS (Real Time Multiple Strip) constituido por 128 detectores de Si colocados en línea, con la longitud activa al máximo. Las medidas se realizaron de $10^{\circ}$ a $70^{\circ}(2 \theta)$ durante 2 horas. El tubo trabajó a $45 \mathrm{kV}$ y $35 \mathrm{~mA}$ y la muestra se giró durante la medida a 16 r.p.m.

Todos los difractogramas fueron analizados con la metodología de Rietveld (13-15).

\subsection{Calorimetría isoterma de conducción}

Se prepararon dos cementos belíticos blancos para realizar un estudio con calorimetría isoterma de conducción. Se seleccionaron el clínker sin activar BB_Ref y el clínker activado con 2,0\% de $\mathrm{Na}_{2} \mathrm{O}, \mathrm{BB} \_2.0 \mathrm{~N}$, y se añadió un $6,8 \%$ y un $11,0 \%$ (en peso) de yeso puro, respectivamente (16). Se utilizaron un cemento Portland blanco comercial tipo I-52.5-R, CC_blanco, y un cemento gris comercial tipo I-52,5-R, CC_gris, para comparar sus calores de hidratación con los de los cementos belíticos blancos de laboratorio. Se midió el calor de hidratación con un calorímetro isotermo de conducción de 8 canales (Thermal Activity Monitor, TAM) de Thermometrics usando ampollas de vidrio. Se prepararon pastas con relación agua/cemento 0,5 , y se midió durante $144 \mathrm{~h}$ a $20^{\circ} \mathrm{C}$.

\section{RESULTADOS Y DISCUSIÓN}

\subsection{Análisis mineralógico}

En la Figura 1 se muestra la zona más representativa de los difractogramas de DRXLP de los clínkeres belíticos blancos de laboratorio, BB_Ref, BB_1.0K, BB_2.0K, BB_1.0N y BB_2.0N.

Los clínkeres belíticos blancos son sistemas más sencillos que los clínkeres Portland grises, ya que contienen tan sólo tres fases principales, $\mathrm{C}_{2} \mathrm{~S}, \mathrm{C}_{3} \mathrm{~S}$ y $\mathrm{C}_{3} \mathrm{~A}$. Su activación con $\mathrm{Na}_{2} \mathrm{O}$ y $\mathrm{K}_{2} \mathrm{O}$ ha generado la estabilización de los polimorfos de alta temperatura del $\mathrm{C}_{2} \mathrm{~S}$. En la Tabla 2 se muestran los análisis cuantitativos de fases de los clínkeres belíticos blancos preparados, además se dan las referencias bibliográficas de las estructuras cristalinas utilizadas. También se muestra el análisis mineralógico de los cementos comerciales que se han utilizado, uno blanco y otro gris, ambos tipo I-52,5-R.
Research Support Services (SCAI) on a PANalytical X'Pert PRO MFD diffractometer with automatic wafer loading and a revolving sample holder. The instrument is likewise fitted with A Johansson-type Ge (111) crystal primary monochromator for pure CuK $\alpha_{1}$ radiation. The divergence and anti-divergence slits were set at $1 / 2$ degree and 0.04 rad. Soller (incident and diffracted beam) slits were used. The detection system consisted in a Real Time Multiple Strip (RTMS) X'Celerator with $128 \mathrm{Si}$ detectors operating in scanning mode and at the maximum active length. The scanning range, from 100 to $70^{\circ}(2 \theta)$, was covered in a two-hour period. The tube operated at $45 \mathrm{kV}$ and $35 \mathrm{~mA}$ and the sample revolved at 16 r.p.m. during the scan.

All the diffractograms were quantified using the Rietveld method (13-15).

\subsection{Isothermal conduction calorimetry}

The white belite cements were prepared for isothermal conduction calorimetric analysis. Pure gypsum was added to both the non-activated clinker BB_Ref $(6.8 \%$ by $w t)$ and the clinker activated with $2.0 \% \mathrm{Na}_{2} \mathrm{O}$, BB_2.ON (11.0\% by wt) (16). The heat of hydration values for these white belite laboratory cements were compared to the values found for commercial white (CC_white) and grey (CC_grey) I-52.5-R Portland cements. Heat of hydration was measured with an eightchannel Thermometrics thermal activity monitor (TAM), using glass vials. A water/cement ratio of 0.5 was used to prepare the pastes, which were monitored for $144 \mathrm{~h}$ at $20^{\circ} \mathrm{C}$.

\section{RESULTS AND DISCUSSION}

\subsection{Mineralogical analysis}

Figure 1 shows the most representative range of the $\angle P X R D$ patterns for white belite laboratory clinkers $B B \_R e f, B B \_1.0 K, B B \_2.0 K, B B \_1.0 N$ and BB_2.0N.

White belite clinkers are simpler than grey Portland clinker systems, having only three main phases: $C_{2} S, C_{3} S$ and $\mathrm{C}_{3} A$. Their activation with $\mathrm{Na}_{2} \mathrm{O}$ and $\mathrm{K}_{2} \mathrm{O}$ stabilized the high temperature $\mathrm{C}_{2} S$ polymorphs. Table 2 gives the quantitative analysis of the phases found in the white belite clinkers prepared, along with bibliographic references for the crystalline structures used. The mineralogical analysis of the white and grey $I-52.5-R$ commercial cements used is likewise shown. 


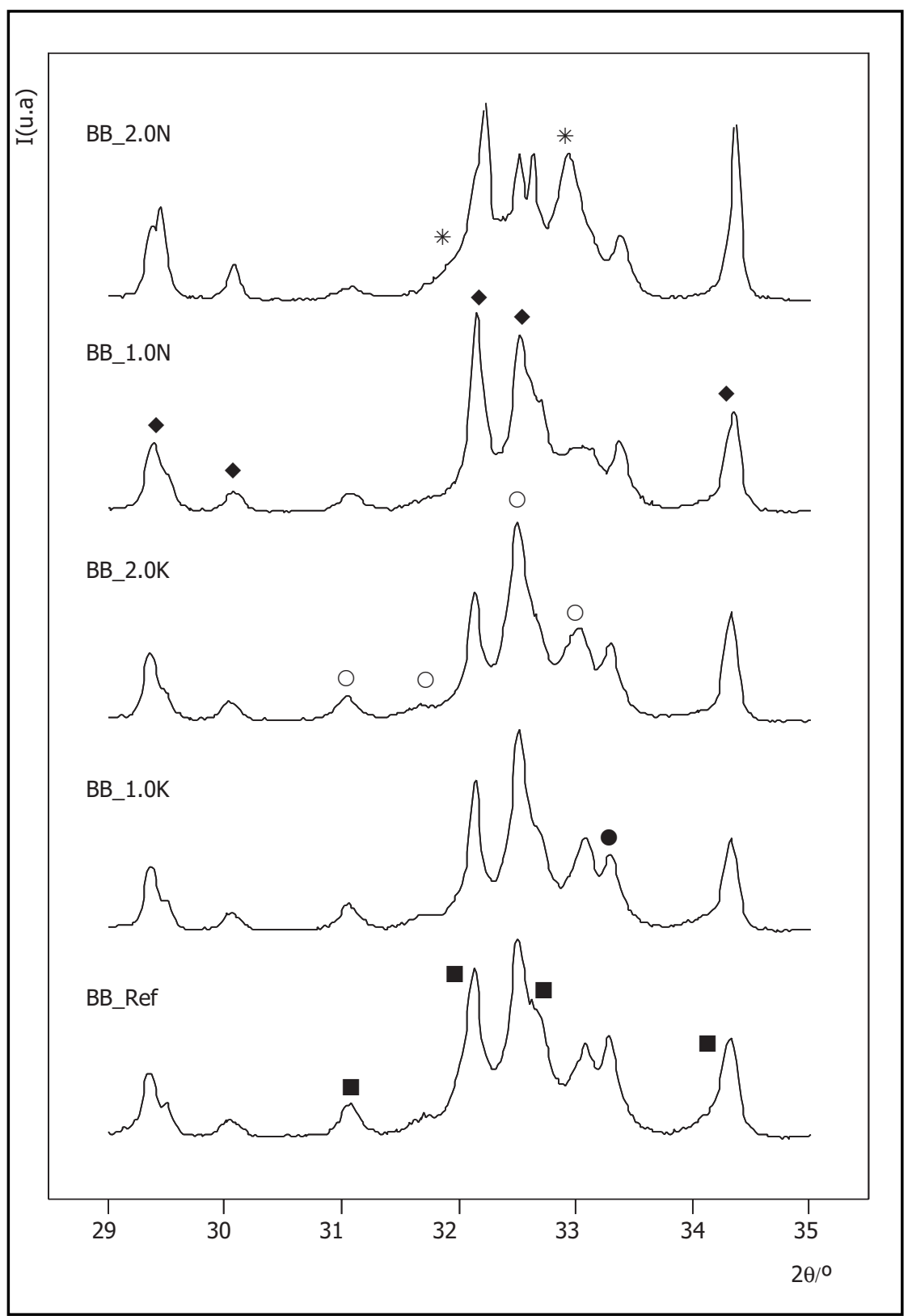

Figura 1. Región de 29 a $35^{\circ}$ (20) de los difractogramas de DRXLP de los clínkeres belíticos blancos preparados. Se han etiquetado picos debidos a las fases: $\beta-\mathrm{C}_{2} \mathrm{~S}$ (cuadrado relleno); $\alpha^{\prime}{ }_{\mathrm{H}}-\mathrm{C}_{2} \mathrm{~S}$ (círculo vacío); $\alpha-\mathrm{C}_{2} \mathrm{~S}$ (estrella); $\mathrm{C}_{3} \mathrm{~S}$ (rombo) y $\mathrm{C}_{3} \mathrm{~A}$ (círculo lleno).

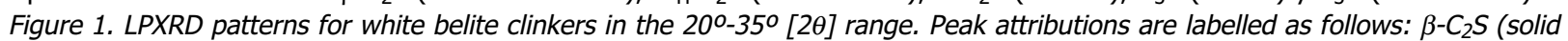
squares); $\alpha^{\prime}{ }^{-} C_{2} S$ (hollow circles); $\alpha-C_{2} S$ (asterisks); $C_{3} S$ (diamonds) and $C_{3} A$ (solid circles).

De los análisis cuantitativos de fases, Tabla 2, se debe destacar la ausencia en todos los clínkeres belíticos de laboratorio de $\gamma-C_{2} S$ y de $C_{4} A F$. La ausencia del primero se debe al enfriamiento rápido y la presencia de iones extraños en las materias primas, lo que impide la transformación polimórfica $\beta \rightarrow \gamma$. La presencia de la forma $\gamma-\mathrm{C}_{2} \mathrm{~S}$ en los cementos belíticos es indeseable por ser hidráulicamente inactiva. Por otro lado, no ha cristalizado el $\mathrm{C}_{4} \mathrm{AF}$ por el pequeño contenido en hierro en las materias primas. Cabe destacar que todos los clínkeres belíticos preparados contienen la fase $\beta-C_{2} S$, que se
Note that according to Table 2, none of the laboratory belite clinkers contained $\gamma-C_{2} S$ or $C_{4} A F$. The absence of the former, due to the rapid cooling process and the presence of foreign ions in the prime materials, prevented the transformation from the beta to the gamma polymorph. The presence of $\gamma-C_{2} S$ in belite cements is undesirable because it is hydraulically inactive. The $C_{4} A F$, in turn, failed to crystallize due to the low iron content in the prime materials. All the belite clinkers prepared contained $\beta-C_{2} S$, however, which stabilizes with rapid cooling and in the presence of 
estabiliza con el enfriamiento rápido y la presencia de iones extraños. La adición de los alcalinos a los crudos ha favorecido la estabilización de $\alpha^{\prime} \mathrm{H}^{-} \mathrm{C}_{2} \mathrm{~S}$ en todos los clínkeres. Al aumentar el contenido de $\mathrm{Na}_{2} \mathrm{O}$ y/o de $\mathrm{K}_{2} \mathrm{O}$, el porcentaje de $\alpha^{\prime} \mathrm{H}^{-} \mathrm{C}_{2} \mathrm{~S}$ aumenta, con la disminución del contenido en $\beta-\mathrm{C}_{2} \mathrm{~S}$. La estabilización de la forma $\alpha$ (de más alta temperatura) se ha conseguido únicamente con la adición a los crudos de un 2,0\% (en peso) de $\mathrm{Na}_{2} \mathrm{O}$ o $\mathrm{K}_{2} \mathrm{O}$. Esta fase se ha estabilizado en cantidades menores a las obtenidas en clínkeres belíticos grises con las mismas cantidades de alcalinos añadidas (9). Esto es debido principalmente al aumento de la tasa de volatilización de los alcalinos con el aumento de la temperatura, ya que los clínkeres belíticos blancos han sido preparados a $1.450{ }^{\circ} \mathrm{C}$, mientras que los clínkeres belíticos grises se prepararon a $1.365^{\circ} \mathrm{C}$. El aumento en el contenido de alcalinos favorece la estabilización del polimorfo monoclínico $\mathrm{C}_{3} \mathrm{~S}_{\mathrm{M} 3}$. El polimorfo ortorrómbico del $\mathrm{C}_{3} \mathrm{~A}$ se ha estabilizado tan sólo en los clínkeres activados con $\mathrm{Na}_{2} \mathrm{O}$. Esto es debido a que el potasio tiene mayor tasa de volatilización que el sodio y a la ausencia del hierro. La presencia de un porcentaje pequeño de $\mathrm{CaO}$ libre en BB_2.0K y BB_2.0N se puede deber a la ausencia de $\mathrm{Fe}_{2} \mathrm{O}_{3}$, que actuaría como fundente y en consecuencia facilitaría el proceso de clinkerización. Aunque el $\mathrm{K}_{2} \mathrm{O}$ y $\mathrm{Na}_{2} \mathrm{O}$ rebajan la temperatura de formación de la fase líquida, en ausencia de $\mathrm{SO}_{3}$ aumentan su viscosidad (26). Hay que indicar que la viscosidad tiene una gran influencia sobre la velocidad de difusión atómica necesaria para formar el $\mathrm{C}_{3} \mathrm{~S}$, que es la última fase formada en el proceso de clinkerización (27). foreign ions. The addition of alkalis to the raw mixes favoured $\alpha^{\prime}{ }_{H}-C_{2} S$ stabilization in all the clinkers. When the $\mathrm{Na}_{2} \mathrm{O}$ and/or $\mathrm{K}_{2} \mathrm{O}$ content rose, the percentage of $\alpha_{H}^{\prime}-C_{2} S$ likewise increased, while the $\beta-C_{2} S$ content declined. The $\alpha$ (the highest temperature) form was stabilized only when $2.0 \%$ (wt) $\mathrm{Na}_{2} \mathrm{O}$ or $\mathrm{K}_{2} \mathrm{O}$ was added to the raw mixes. For any given percentage of alkaline addition, the proportion of this phase that was stabilized was lower in the grey than in the white belite cements (9). This was due primarily to the increase in alkali volatilization with rising temperatures, for the white belite clinkers were kilned to $1,450{ }^{\circ} \mathrm{C}$ but the grey belite clinkers to only $1,365^{\circ} \mathrm{C}$. Increasing the alkaline content favoured the stabilization of the monoclinic polymorph $C_{3} S_{M 3}$. The orthorhombic polymorph $\mathrm{C}_{3} \mathrm{~A}$ was stabilized in the $\mathrm{Na}_{2} \mathrm{O}$ activated clinkers only because potassium has a higher volatilization rate than sodium, and due to the absence of iron. The presence of a small percentage of free lime in BB_2.0K and BB_2.0N may have been the result of the absence of $\mathrm{Fe}_{2} \mathrm{O}_{3}$, which would act as a flux, thereby favouring clinkerization. Although $\mathrm{K}_{2} \mathrm{O}$ and $\mathrm{Na}_{2} \mathrm{O}$ lowered the temperature at which the liquid phase formed, in the absence of $\mathrm{SO}_{3}$ they raised its viscosity (26). Viscosity, in turn, affects the rate of atomic diffusion needed to form $C_{3} S$, the last phase to appear in clinkerization (27).

Tabla 2 / Table 2

Composición mineralógica (\% en peso) de los clínkeres belíticos blancos y de los cementos comerciales a partir de los datos de DRXLP. Rietveld quantitative análisis findings for white belite clinkers and comercial cements.

\begin{tabular}{|c|c|c|c|c|c|c|c|c|c|c|c|c|}
\hline & $\begin{array}{c}\alpha-\mathbf{C}_{2} \mathbf{S} \\
\%\end{array}$ & $\begin{array}{c}\alpha_{H}^{\prime}-C_{2} S \\
\%\end{array}$ & $\begin{array}{c}\beta-C_{2} S \\
\%\end{array}$ & $\begin{array}{l}\mathrm{C}_{3} \mathrm{~S}_{\mathrm{T}} \\
\%\end{array}$ & $\begin{array}{c}\mathrm{C}_{3} \mathrm{~S}_{\mathrm{M3}} \\
\%\end{array}$ & $\begin{array}{c}\mathrm{C}_{3} \mathrm{~A}_{\mathrm{CII}} \\
\%\end{array}$ & $\begin{array}{c}C_{3} A_{\text {ort }} \\
\%\end{array}$ & $\begin{array}{c}\mathbf{C}_{4} \mathbf{A F} \\
\%\end{array}$ & $\begin{array}{c}\mathrm{CaO} \\
\%\end{array}$ & $\begin{array}{c}\text { Yeso } \\
\%\end{array}$ & $\begin{array}{c}\text { Basanita } \\
\%\end{array}$ & $\begin{array}{c}\text { RWp } \\
\%\end{array}$ \\
\hline BB_Ref & - & $28.0(3)$ & $36.7(3)$ & $24.3(2)$ & - & $11.0(2)$ & - & - & - & - & - & 6.8 \\
\hline BB_1.0K & - & $35.6(2)$ & $26.3(3)$ & 17.1(5) & $10.8(5)$ & $10.2(2)$ & - & - & - & - & - & 7.1 \\
\hline BB_2.0K & $3.9(3)$ & $41.0(3)$ & $17.2(4)$ & $14.5(6)$ & $13.9(6)$ & $8.9(2)$ & - & - & $0.60(5)$ & - & - & 6.9 \\
\hline BB_1.0N & - & $31.6(3)$ & $25.3(4)$ & $21.8(6)$ & $10.6(7)$ & $4.5(2)$ & $6.2(2)$ & - & - & - & - & 6.8 \\
\hline BB_2.0N & $16.4(4)$ & $16.7(8)$ & $23.2(6)$ & $6.0(6)$ & $26.7(5)$ & - & $9.5(2)$ & - & $1.50(5)$ & - & - & 8.9 \\
\hline CC_blanco & - & - & $22.3(3)$ & - & $68.7(1)$ & $3.0(1)$ & - & - & - & $2.9(1)$ & 3.1(1) & 8.0 \\
\hline CC_gris* & - & - & 13.5() & - & $65.5(2)$ & $4.4(2)$ & - & $10.6(3)$ & - & - & $3.6(1)$ & 7.9 \\
\hline $\begin{array}{l}\text { Ref. bibliog. / } \\
\text { Bibliog. Ref. }\end{array}$ & (17) & (17) & (17) & (18) & (19) & (20) & (20) & (21) & (22) & (23) & (24) & \\
\hline
\end{tabular}

* Contiene además $1,1(1) \%$ en peso de $\mathrm{MgO}(25)$, y menos de $0,5 \%$ en peso de $\mathrm{SiO}_{2}$ y sulfatos alcalinos / Also contains 1.1 (1)\% (wt) MgO (25) and less than $0.5 \%$ (wt) $\mathrm{SiO}_{2}$ and alkaline sulphates.

En la Figura 2 se muestran, a modo de ejemplo, los difractogramas ajustados con el método de Rietveld de los clínkeres (a) BB_Ref, (b) BB_2.0K y (c) BB_2.0N, respectivamente.
Figure 2 shows the Rietveld-refined diffractograms for clinkers (a) BB_Ref, (b) BB_2.0K and (c) BB_2.0N, by way of example. 


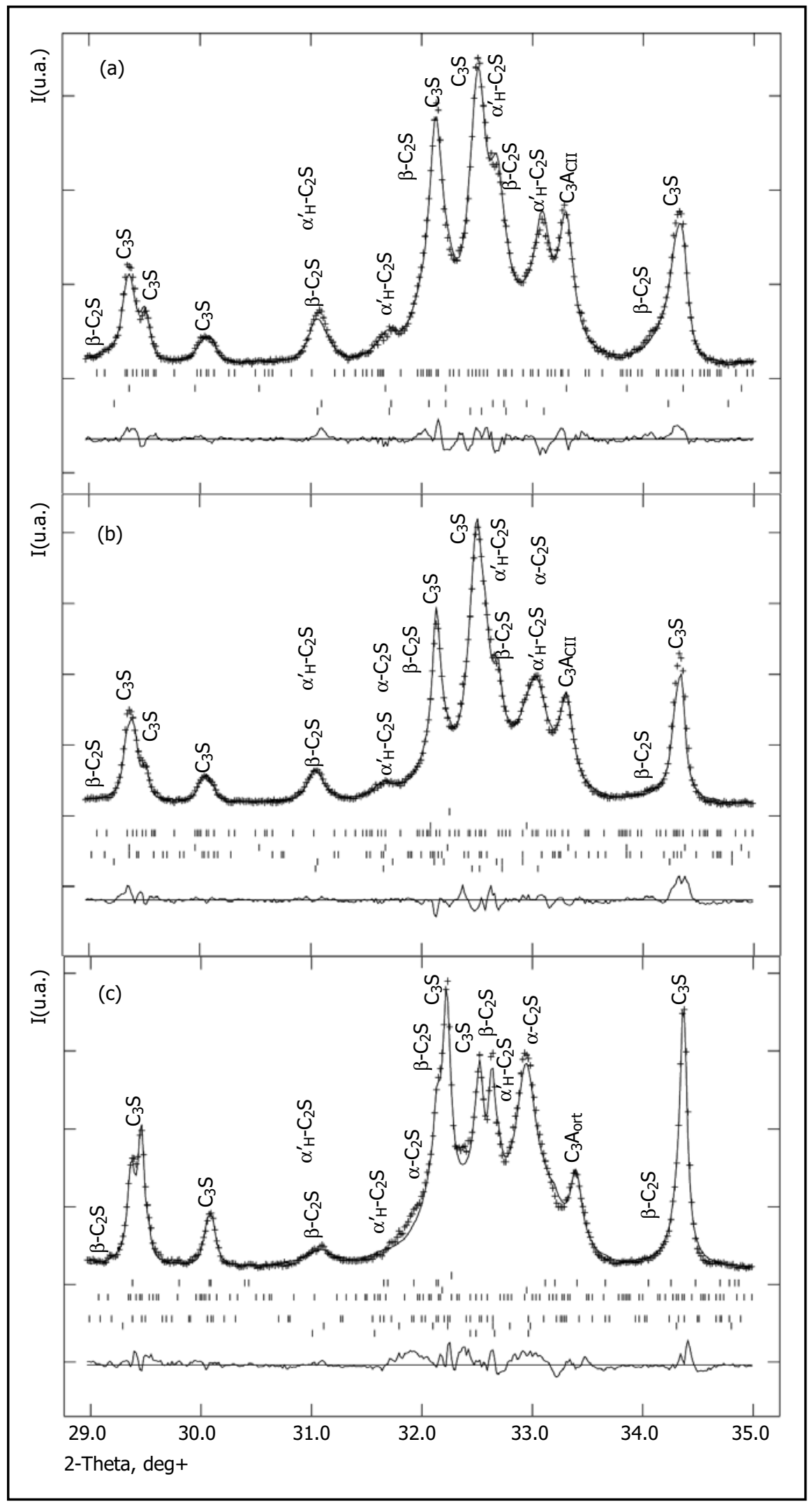

Figura 2. Región de 29 a $35^{\circ}$ (2ө) de los difractogramas de DRXLP ajustados con el método de Rietveld de: (a) BB_Ref, (b) BB_2.0K y (c) BB_2.0N. Se han etiquetado los picos debidos a cada fase.

Figure 2. $29^{\circ}-35^{\circ}$ (20) range of Rietveld-refined LPXRD patterns for: (a) BB_Ref, (b) BB_2.0K and (c) BB_2.0N. Peak attributions labelled. 


\subsection{Efecto de volatilización de $\mathrm{K}_{2} \mathrm{O}$ y $\mathrm{Na}_{2} \mathrm{O}$}

En la Tabla 3 se muestran los porcentajes en peso de $\mathrm{K}_{2} \mathrm{O}$ y $\mathrm{Na}_{2} \mathrm{O}$ en los clínkeres belíticos blancos determinados por espectroscopía de emisión atómica y también sus porcentajes de volatilización.

\subsection{Effect of $\mathrm{K}_{2} \mathrm{O}$ and $\mathrm{Na}_{2} \mathrm{O}$ volatilization}

Table 3 gives the percentages (wt) of $\mathrm{K}_{2} \mathrm{O}$ and $\mathrm{Na}_{2} \mathrm{O}$ in the white belite clinkers as determined by atomic emission spectroscopy, along with the volatilization percentages.

Tabla 3 / Table 3

Porcentaje en peso de sodio y potasio, expresadas en forma de sus óxidos, en los clínkeres belíticos blancos determinados por espectroscopía de emisión atómica. Además, se muestra el porcentaje de volatilización. Percentage (wt) of sodium and pottasium oxides in white belite clinkers measured by atomic emisión spectroscopy. Volatilization percentages also shown.

\begin{tabular}{|c|c|c|c|c|}
\hline & $\mathrm{K}_{2} \mathrm{O} / \%$ & $\begin{array}{c}\% \mathbf{K}_{2} \mathrm{O} \text { volatilizado / } \\
\text { volatized }\end{array}$ & $\mathrm{Na}_{2} \mathrm{O} / \%$ & $\begin{array}{c}\% \mathrm{Na}_{2} \mathrm{O} \text { volatilizado / } \\
\text { volatized }\end{array}$ \\
\hline BB_Ref & 0.01 & 95 & 0.20 & $* *$ \\
\hline BB_1.0K & 0.13 & 87 & 0.17 & $* *$ \\
\hline BB_2.0K & 0.32 & 84 & 0.19 & ** \\
\hline BB_1.0N & 0.00 & 100 & 0.74 & 26 \\
\hline BB_2.0N & 0.02 & 91 & 1.30 & 35 \\
\hline
\end{tabular}

** No había pérdida / No loss.

Del análisis de la Tabla 3 se puede concluir que la tasa de volatilización de $\mathrm{K}_{2} \mathrm{O}$ es mucho más elevada que la de $\mathrm{Na}_{2} \mathrm{O}$. Como en el caso de los clínkeres belíticos grises $(9,10)$, el potasio retenido en el clínker aumenta al aumentar la cantidad añadida al crudo, aunque el porcentaje de volatilización es más elevada en los clínkeres blancos, debido a que la temperatura de clinkerización es más alta. De hecho, en los clínkeres BB_Ref, BB_1.0N y $\mathrm{BB} \_2.0 \mathrm{~N}$ se ha volatilizado casi la totalidad del $\mathrm{K}_{2} \mathrm{O}$ presente en los crudos. La tasa de volatilización del $\mathrm{Na}_{2} \mathrm{O}$ es mucho menor, cercana al $30 \%$, en las condiciones de preparación de estos clínkeres. En los clínkeres BB_Ref, BB_1.0K y BB_2.0K el contenido de sodio medido es ligeramente mayor que su porcentaje nominal en los crudos, comparar Tablas 1 y 3 . Este desacuerdo se debe muy probablemente a la diferencia entre las técnicas usadas para medir este elemento en las materias y en el clínker final.

\subsection{Calorimetría de conducción isoterma}

Los cementos belíticos blancos se molieron hasta obtener una finura similar, expresada en parámetro Blaine: $430 \mathrm{~m}^{2} / \mathrm{kg}$ el cemento fabricado con el clínker BB_Ref y $420 \mathrm{~m}^{2} / \mathrm{kg}$ el cemento obtenido con BB_2.0N. Los cementos comerciales tenían un finura de 530 $\mathrm{m}^{2} / \mathrm{kg}$ el blanco y de $520 \mathrm{~m}^{2} / \mathrm{kg}$ el gris. En la Figura 3 se muestran las curvas calorimétricas, normalizadas a masa de cemento, de los cuatro cementos estudiados en el rango seleccionado de 0 a $30 \mathrm{~h}$.

Las curvas de calor de hidratación liberado son muy distintas entre ellas y su interpretación en detalle requiere la realización de otros análisis adicionales, como son
According to Table 3, the volatilization rate was much higher in $\mathrm{K}_{2} \mathrm{O}$ than in $\mathrm{Na}_{2} \mathrm{O}$. As has been previously shown for grey belite clinkers $(9,10)$, the potassium retained in the clinker grew with rising percentages of the addition. The volatilization rate was higher in white clinkers, however, due to the higher clinkerization temperature. In clinkers BB_Ref, BB_1.ON and BB_2.0N, nearly all the $K_{2} O$ present in the raw mixes volatilized. Under the conditions in which these clinkers were prepared, the $\mathrm{Na}_{2} \mathrm{O}$ volatilization rate was much lower, at around $30 \%$. In clinkers BB_Ref, BB_1.0K and $B B \_2.0 K$, the sodium content measured was slightly higher than the nominal percentage in the raw mixes (compare Tables 1 and 3). This discrepancy was very likely due to the use of different techniques to measure this element in the materials and the end product.

\subsection{Isothermal conduction calorimetry}

White belite cements were ground to similar Blaine fineness values: $430 \mathrm{~m}^{2} / \mathrm{kg}$ for the cement made with BB_Ref clinker and $420 \mathrm{~m}^{2} / \mathrm{kg}$ for the cement made with $B B \_2.0 N$. The Blaine fineness values for the commercial cements were $530 \mathrm{~m}^{2} / \mathrm{kg}$ (white) and $520 \mathrm{~m}^{2} / \mathrm{kg}$ (grey). Figure 3 shows the heat flow curves, normalized to cement mass, for the four cements studied in the selected range: 0 to $30 \mathrm{~h}$.

The heat of hydration curves clearly varied. Full interpretation would require further analyses, such as in situ X-ray diffraction heat of hydration studies. The total 


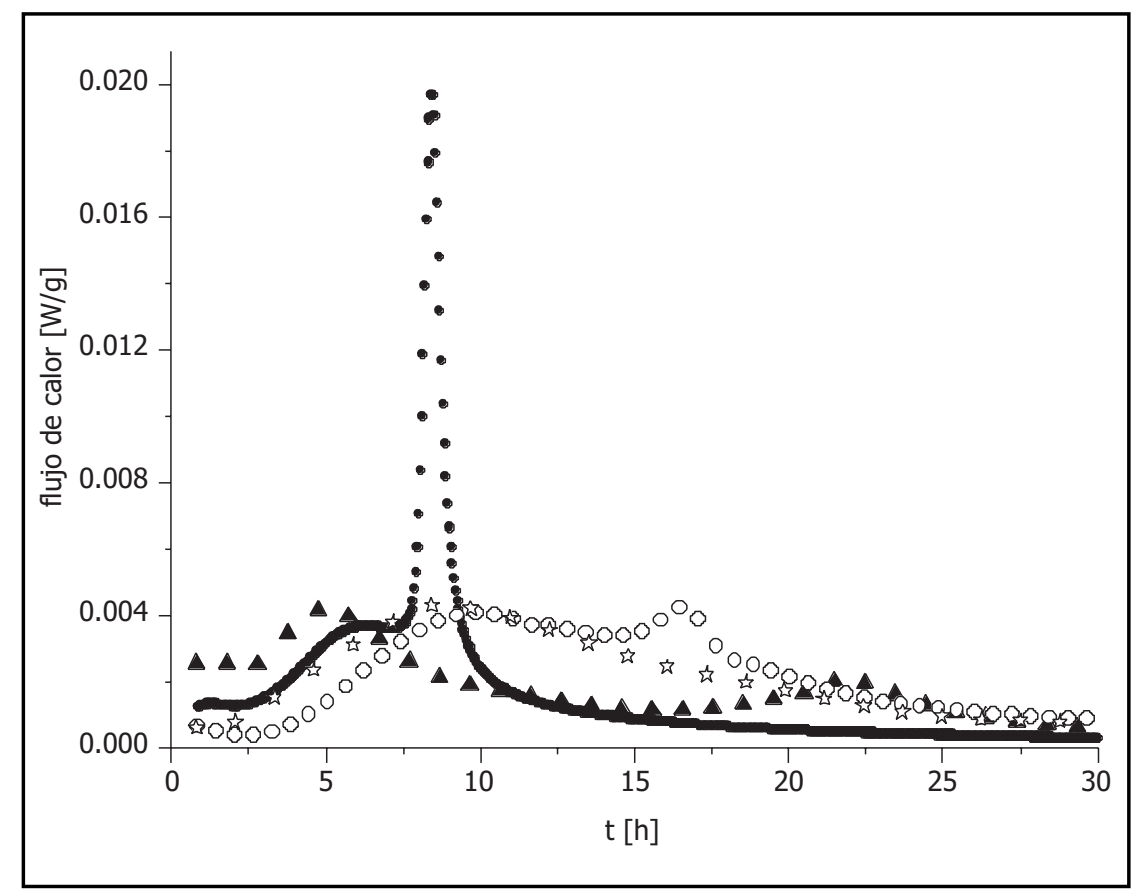

Figura 3. Curvas calorimétricas, normalizadas a masa de cemento, de las pastas estudiadas (relación agua/cemento: 0,50): BB_Ref (puntos rellenos) y BB_2.0N (triángulos), CC_blanco (estrellas) y CC_gris (círculos vacíos).

Figure 3. Heat flor curves for the pastes studied normalizad to cement mass (water/cement ratio: 0.50): BB-Ref (solid circles), BB_2.0N (triangles), CC_white (stars) and CC_grey (hollow circles).

estudios de hidratación in-situ utilizando difracción de rayos-X. En la Tabla 4 se dan los calores totales liberados por los cuatro cementos a distintos tiempos.

Analizando los datos de la Tabla 4, cabe destacar que los cementos belíticos liberan más calor que los cementos comerciales en las primeras 5 h. Esta liberación de calor inicial se debe a la formación de ettringita a partir de la reacción del yeso con los aluminatos (26). Al ser mayor el porcentaje de $\mathrm{C}_{3} \mathrm{~A}$ en los cementos belíticos blancos, era de esperar este fenómeno. Sin embargo, a partir de las 24 h de hidratación, los cementos belíticos, incluyendo el cemento activado, liberan menos calor que los comerciales. La adición de alcalinos a los clínkeres belíticos ha favorecido la estabilización de diversas cantidades de los polimorfos de alta temperatura de la belita, sin embargo con estos resultados no se ha podido determinar que estas formas liberen más calor de hidratación en la escala de tiempo estudiada.

Se ha seleccionado el clínker belítico con 2,0\% de $\mathrm{Na}_{2} \mathrm{O}$, puesto que contiene aprox. $16 \%$ en peso de $\alpha-\mathrm{C}_{2} \mathrm{~S}$, Tabla 2. Este cemento ha ido liberando más calor al hidratarse que el cemento belítico de referencia hasta las $72 \mathrm{~h}$. A las $144 \mathrm{~h}$, ambos materiales han desarrollado el mismo calor de hidratación. La influencia de la cantidad de yeso añadida en este comportamiento se está estudiando en la actualidad. También se está estudiando actualmente la correlación entre la liberación de calor y el desarrollo de resistencias mecánicas. heat released by the four cements at different experimental times are given in Table 4.

Further to the data in Table 4, in the first five hours the belite cements released more heat than the commercial cements. This initial heat release, generated by ettringite formation resulting from the gypsum - aluminate reaction (26), was the behaviour expected, given the higher percentage of $C_{3} A$ in white belite cements. After $24 \mathrm{~h}$ of hydration, however, the belite cements, including the activated cement, released less heat than the commercial materials. Adding alkalis to the belite clinkers favoured the stabilization of several amounts of high temperature polymorphs, although with the present findings these forms could not be shown to have released greater heat of hydration in the time frame studied.

The belite clinker chosen was the sample activated with $2.0 \% \mathrm{Na}_{2} \mathrm{O}$, for its high $\alpha-\mathrm{C}_{2} \mathrm{~S}$ content (approximately $16 \%(w t))$ (Table 2). This cement released more heat of hydration than the reference cement up to $t=72 \mathrm{~h}$. After $144 h$, both materials released the same total heat of hydration. How this behaviour may have been impacted by the amount of gypsum added is presently being researched, along with the correlation between heat flow and the development of mechanical strength. 
Tabla 4 / Table 4

Calor total liberado $(\mathrm{J} / \mathrm{g})$ en función del tiempo, normalizado a la masa de cemento, en la hidratación de las pastas estudiadas. Total head released $(\mathrm{J} / \mathrm{g})$, normalizad to cement mass, in paste hydration.

\begin{tabular}{|c|c|c|c|c|c|}
\hline & $\mathbf{5 ~ h}$ & $\mathbf{2 4} \mathbf{~ h}$ & $\mathbf{4 8} \mathbf{~ h}$ & $\mathbf{7 2} \mathbf{~ h}$ & $\mathbf{1 4 4} \mathbf{~ h}$ \\
\hline CBB_Ref $[\mathrm{J} / \mathrm{g}]$ & 27 & 172 & 196 & 210 & 249 \\
\hline CBB_2.0N $[\mathrm{J} / \mathrm{g}]$ & 46 & 172 & 216 & 230 & 252 \\
\hline CC_blanco $[\mathrm{J} / \mathrm{g}]$ & 10 & 213 & 275 & 296 & 300 \\
\hline CC_gris $[\mathrm{J} / \mathrm{g}]$ & 20 & 210 & 268 & 300 & 330 \\
\hline
\end{tabular}

\section{CONCLUSIONES}

Se han activado clínkeres belíticos blancos estabilizando las formas de alta temperatura del silicato dicálcico, $\alpha_{H}^{\prime}$ y $\alpha$, con la adición de pequeñas cantidades de sodio o potasio. Los clínkeres con mayores contenidos en alcalinos presentan mayores porcentajes en peso de las formas de alta temperatura del $\mathrm{C}_{2} \mathrm{~S}$. Sin embargo, debido a la mayor tasa de volatilización del potasio, en estas condiciones, el mayor porcentaje de fase $\alpha-\mathrm{C}_{2} \mathrm{~S}$ se ha obtenido con un $2,0 \%$ en peso de $\mathrm{Na}_{2} \mathrm{O}$. Los estudios calorimétricos confirman que hasta las primeras $72 \mathrm{~h}$ el cemento belítico activado libera más calor que el cemento sin activar. Sin embargo, los cementos belíticos no alcanzan el calor liberado a $144 \mathrm{~h}$ por un cemento blanco alítico (rico en $\mathrm{C}_{3} \mathrm{~S}$ ) comercial.

\section{AGRADECIMIENTOS}

Se agradece la financiación a través del proyecto de excelencia de la Junta de Andalucía (P06-FQM-01328). Además, agradecemos al Dr. Luís Miguel Ordóñez su ayuda en la realización del estudio calorimétrico, así como sus comentarios en la interpretación de los mismos. Por otro lado, se agradece a la Dr. Laura León-Reina por la toma de datos de difracción de rayos-X.

\section{CONCLUSIONS}

The activation of white belite clinkers with small amounts of sodium or potassium was shown to stabilize the high temperature forms of dicalcium silicate, $\alpha_{H}^{\prime}$ and $\alpha$. The clinkers with greater alkaline contents had larger percentages (wt) of high temperature $\mathrm{C}_{2} \mathrm{~S}$ polymorphs. Nonetheless, due to the higher rate of potassium volatilization under the experimental conditions, the highest percentage of phase $\alpha-C_{2} S$ was obtained with $2.0 \%$ (wt) $\mathrm{Na}_{2} \mathrm{O}$. Calorimetric studies confirmed that for the first $72 h$, the activated belite cement released more heat than the non-activated cement. After $144 h$, however, the belite cements released less heat than an alite $\left(C_{3} S\right.$-rich) commercial cement.

\section{ACKNOWLEDGEMENTS}

This study was funded by the Regional Government of Andalusia under excellence in research grant (P06-FQM01328). The authors wish to thank $\mathrm{Dr}$ Luis Miguel Ordóñez for his assistance in conducting the calorimetric study and interpreting the findings. Dr Laura León-Reina is likewise thankfully acknowledged for the X-ray diffraction readings.

\section{BIBLIOGRAFÍA / BIBLIOGRAPHY}

(1) Lukasik, J.; Damtoft, J.S.; Herfort, D.; Sorrentino, D.; Gartner, E.M.: "Sustainable Development and Climate Change Initiatives". 12th International Congress on the Cement Chemistry, Montreal, ST3 (2007), MPL-1.

(2) Gartner, E.: "Industrially Interesting Approaches to «Low-CO2» Cements," Cem. Concr. Res., vol. 34[9] (2004), pp. $1489-1498$.

(3) Popescu, C.D.; Muntean, M.; Sharp, J.H.: "Industrial Trial Production of Low Energy Belite Cement", Cem. Concr. Composites, vol. 25(7) (2003), pp 689-693. doi:10.1016/S0958-9465(02)00097-5

(4) Mehta, P.K.: "Investigation on Energy-Saving Cements", World cement technology, vol. 11(5) (1980), pp. 166-177.

(5) Pajares, I.; De la Torre, A. G.; Martínez-Ramírez, S.; Puertas, F.; Blanco-Varela, M. T.; Aranda, M.A.G.: "Quantitative Analysis of Mineralised White Portland Clinkers: the structure of Fluorellestadite". Powder Diffraction, vol. 17 (2002), pp. $281-286$. doi:10.1154/1.1505045

(6) Muntean, M.: "White belitic Portland cement" 9th International Congress on the cement chemistry, New Dheli, vol. 3 (1992), pp. 4550.

(7) Chatterjee, A.K.: "High Belite Cements. Present Status and Future Technological Options: Part I". Cem. Concr. Res. Vol. 26 (1996), pp. 1213-1225.

(8) Gies, A.; Knofel, D.: "Influence of alkalies on the composition of belite-rich cement linkers and the technological properties of the resulting cements". Cem. Concr. Res., Vol. 16 (1986), pp. 411-422. doi:10.1016/0008-8846(86)90117-1 
(9) Morsli, K.; De la Torre, A. G.; Stöber, S.; Cuberos, A. J. M.; Zahir, M.; Aranda, M. A. G.: "Quantitative Phase Analysis of Laboratory Active Belite Clinkers by Synchrotron Powder Diffraction". J. Amer. Ceram. Soc., vol. 90(10) (2007), pp. 3205-3212.

(10) Morsli, K.; De la Torre, A. G.; Zahir, M.; Aranda, M. A. G.: "Mineralogical Phase Analysis of Alkali and Sulfate Bearing Belite Rich Laboratory Clinkers" Cem. Concr. Res., vol. 37 (2007), pp. 639-646. doi:10.1016/j.cemconres.2007.01.012

(11) Chatterjee, A.K.: "Special Cements", en Structure and Performance of Cements. Edited by J. Bensted and P. Barnes, Spon Spress, London y New York, 2002, pp. 186-236.

(12) Gies, A.; Knofel, D.: "Influence of sulfur on the Composition of Belite-Rich Cement Clinkers and the Technological Properties of the Resulting Cements", Cem. Concr. Res., vol. 17(2) (1987), pp. 317-328. doi:10.1016/0008-8846(87)90114-1

(13) Rietveld, H. M.: "A profile refinement method for nuclear and magnetics structures". J. Applied Crystallog., vol. 2 (1969), pp. 6571. doi: $10.1107 / S 0021889869006558$

(14) De la Torre, A. G.; Cabeza, A.; Calvente, A.; Bruque, S.; Aranda, M. A. G.: "Full Phase Analysis of Portland Clinker by Penetrating Synchrotron Powder Diffraction" Analytical Chemistry, vol. 73[2] (2001)15, pp. 1-156.

(15) De la Torre, A. G.; Aranda, M. A. G.: "Accuracy in Rietveld quantitative phase analysis of Portland cements", J. Applied Crystallog., vol. 36 (5) (2003), pp. 1169-1176.

(16) Haskell, W. E.: "Three factors govern optimum gypsum content of cement", Rock products, vol. April (1959), pp. 108-149.

(17) Mumme, W. G.; Hill, R. J.; Bushnell-Wye, G.; Segnit, E. R.: "Rietveld structure refinement, crystal chemistry and calculated powder diffraction data for the polymorphs of dicalcium silicate and related phases", N. Jb. Miner. Abh., vol. 169 (1995), pp. 35-68.

(18) Golovastikov, N. I.; Matveeva, R. G.; Belov, N. V.: "Crystal structure of the tricalcium silicate $3 \mathrm{CaO} \mathrm{SiO}_{2}=\mathrm{C}_{3} \mathrm{~S}^{\prime}$, Sov. Phys. Crystallog., vol. 20[4], (1975), pp. 441-445.

(19) De la Torre, A. G.; Bruque, S.; Campo, J.; Aranda, M. A. G.: "The superstructure of $C_{3} S$ from synchrotron and neutron powder diffraction and its role in quantitative phase analyses", Cem. Concr. Res., vol. 32[9] (2002), pp. 1347-1356.

(20) Takeuchi, Y.; Nishi, F.; Maki, I.: "Crystal-chemical characterization of the $3 \mathrm{CaO} \bullet \mathrm{Al}_{2} \mathrm{O}_{3}-\mathrm{Na}_{2} \mathrm{O}$ solid-solution series", Z. Kristallog., vol. 152 [3-4] (1980), pp. 259-307.

(21) Colville, A. A.; Géller, S.: "The crystal structure of brownmillerite, Ca2FeAlO5", Acta Cryst., vol. B27 [12] (1971), pp. $2311-2315$.

(22) Natta, G.; Passerini, L.: "Soluzioni solide, isomorfismo e simmorfismo tra gli ossidi dei metalli bivalenti. Sistemi: CaO-CdO. CaOMnO, CaO-CoO, CaO-NiO, CaO- MgO", Gazzetta Chimica Italiana, vol. 59 (1929), pp. 129-154.

(23) De la Torre, A. G.; López-Olmo, M-G; Álvarez-Rua, C.; García-Granda, S.; Aranda, M. A. G.: "Structure and Microstructure of Gypsum and its Relevance to Rietveld Quantitative Phase Analyses", Powder Diffraction, vol. 19 (2004), pp. 240-246. doi:10.1154/1.1725254

(24) Bezou, C.; Nonat, A.; Mutin, J. C.; Christensen, A. N.; Lehmann, M. S.: "Investigation of the crystal structure of gamma-CaSO $\mathrm{CaSO}_{4} \cdot 0.5 \mathrm{H}_{2} \mathrm{O}$ and $\mathrm{CaSO}_{4} \cdot 0.6 \mathrm{H}_{2} \mathrm{O}$ by powder diffraction methods". J. Solid State Chemistry, vol. 117 (1995), pp. $165-176$. doi:10.1006/jssc. 1995.1260

(25) Maslen, E. N.; Streltsov, V. A.; Streltsova, N. R.; Ishizawa, N.: "Electron density and optical anisotropy in rhombohedral carbonates. III. Synchrotron X-ray studies of $\mathrm{CaCO}_{3}, \mathrm{MgCO}_{3}$ and $\mathrm{MnCO}_{3}$ ". Acta Crystallog, vol. B51 (1995), pp. 929-939.

(26) Taylor, H. F. W.: "Cement Chemistry". Thomas Telford. London, 1997.

(27) De la Torre, A. G.; Morsli, K.; Zahir, M.; Aranda, M. A. G.: J. Applied Crystallog, vol. 40 (2007), pp. 999-1007. 